\title{
Comment des élèves lisent un document composite en géographie
}

Un modèle d'analyse épistémologique et sémiotique en didactique de la géographie

How students read a geographic composite document. A model of epistemogical and semiotic analysis in the didactis of geography.

Jean-François Thémines

\section{(2) OpenEdition \\ Journals}

Édition électronique

URL : http://journals.openedition.org/pratiques/8416

DOI : $10.4000 /$ pratiques. 8416

ISSN : 2425-2042

Éditeur

Centre de recherche sur les médiations (CREM)

Référence électronique

Jean-François Thémines, "Comment des élèves lisent un document composite en géographie »,

Pratiques [En ligne], 185-186 | 2020, mis en ligne le 30 juin 2020, consulté le 15 octobre 2020. URL

http://journals.openedition.org/pratiques/8416 ; DOI : https://doi.org/10.4000/pratiques.8416

Ce document a été généré automatiquement le 15 octobre 2020.

(ㄷ) Tous droits réservés 


\section{Comment des élèves lisent un document composite en géographie}

Un modèle d'analyse épistémologique et sémiotique en didactique de la géographie

How students read a geographic composite document. A model of epistemogical and semiotic analysis in the didactis of geography.

Jean-François Thémines

\section{Introduction}

1 Les documents ont été précocement ${ }^{1}$ l'objet de recherches en didactiques de l'histoire et de la géographie (Audigier, 1993). «Angle d'attaque privilégié » pour construire une approche didactique de ces disciplines, ils « parcourent et habitent les pratiques de nos disciplines [scolaires] depuis plus d'un siècle » (ibid., p. 11).

2 La géographie scolaire a une longue histoire d'usages d'images de la Terre. Après les cartes dans les années 1870 (Lefort, 1992), les images de paysage au tournant du $\mathrm{xx}^{\mathrm{e}}$ siècle, les films dans les années 1930, les images satellitaires dans les années 1980, les globes virtuels et les outils de géolocalisation et d'imagerie numérique en trois dimensions entrent depuis dix ans dans les pratiques, sans que les autres types d'image n'aient disparu. En dépit de cette accumulation et de cette diversité, l'écriture institutionnelle de compétences (en collège) et de capacités (en lycée) reste très générique. La pluralité et l'association des langages sont présentées comme une nécessité, sans que ne soit appréhendée l'éventuelle complexité de leur apprentissage. C'est ainsi que l'actualité des prescriptions, des recommandations et des pratiques en géographie scolaire rencontre la notion de document composite (Leclaire-Halté \& Maisonneuve, 2018).

Pour des élèves, qu'est-ce que lire et comprendre un document composite en classe de géographie? À quelles difficultés intrinsèques à ce type de document sont-ils confrontés pour interpréter le monde tel qu'il est conçu dans cette discipline scolaire? 
4 Après avoir situé la lecture de document composite dans les questionnements de didactique de la géographie, cet article en propose un modèle d'analyse d'inspiration sémiotique. Illustré pour une séance conduite en classe de quatrième, ce modèle permet d'identifier des sources de difficultés mésestimées concernant la lecture du document composite.

\section{Document, document composite : questionnements en didactique de la géographie}

5 La notion de document en classe de géographie est polysémique pour deux raisons. L'une concerne la géographie scolaire, l'autre l'ensemble des disciplines d'enseignement.

\section{Problématique disciplinaire spécifique : le document pour être de plain-pied au monde}

6 Un résultat important des recherches en didactique de la géographie est le réalisme épistémologique qui caractérise la production de savoirs dans la discipline scolaire (Audigier, 1996 ; Tutiaux-Guillon, 2004).

En enseignant les résultats, en faisant comme si tout cela était vrai, par sa forme même, par les exercices et les évaluations qu'elles mettent en œuvre, l'histoire et la géographie font comme si elles disaient la réalité du monde [...] elles effacent le rôle des langages et des points de vue [...] Elles ignorent le rôle des langages comme producteurs de sens, de manières de penser le monde. (Audigier, 1996, p. 75).

7 Le document fonctionne comme un substitut du réel (Margairaz, 1988). Il participe à «produire l'illusion que les professeurs, les savoirs enseignés et finalement les élèves sont de plain-pied au monde » (Thémines, 2002, p. 48).

8 La géographie scolaire se caractérise par la multimodalité (Nonnon, 2006; Lebrun \& Lacelle, 2012) de ses moyens d'expression. À l'iconographie et à la cartographie sur support papier, se sont ajoutées leurs développements numériques (Genevois, 2016) issues de pratiques professionnelles (géomatique ${ }^{2}$ ) et sociales (géolocalisation et géovisualisation). Les enquêtes datant de l'entrée des globes virtuels (Google Earth, Géoportail) dans les classes montrent que leurs usages se situent, à l'instar des pratiques cartographiques dominantes (Fontanabona, 2000) dans la tradition réaliste de la géographie scolaire. Cet état de fait conduit à poser la dimension épistémologique comme essentielle pour l'analyse de la compréhension de documents composites en géographie par les élèves.

\section{Problématique disciplinaire générique : support, langage et document}

9 En géographie comme dans d'autres disciplines scolaires, trois niveaux d'appréhension du document sont souvent confondus : le « document comme support », le « document comme discours" et le «document comme trace d'une activité et d'attribution de sens » (Nonnon, 2012). 
10 S'agissant de support, c'est-à-dire de matérialité, la notion de document comporte l'idée d'« espace perceptuel» (Leclaire-Halté \& Maisonneuve, 2018). Cette propriété s'applique d'une part au support qui confère une unité au document, et d'autre part, aux fragments qui le composent et empruntent à divers systèmes sémiotiques pour la représentation du réel et l'expression d'un contenu géographique. Le développement de supports numériques en articulation avec des supports papier ouvrent de nouvelles possibilités d'espace perceptuel. Par exemple, la géomatique scolaire permet de disposer en un seul espace perceptuel simultané (l'écran) d'une multiplicité de couches d'informations géolocalisées, qu'il est possible au lecteur d'activer ou désactiver, rendre visibles ou masquer.

11 Concernant le document comme discours, il apparaît que le document composite en géographie est constitué de " composants relevant de systèmes sémiotiques de nature différente, des textes, des images, des graphiques, des cartes...» (Nonnon, 2012.). Les usages des photographies et des cartes ont fait l'objet d'analyses didactiques (Fontanabona, 2000 ; Mendibil, 2008) qui ont montré la spécificité des langages qui les fondent. Ces langages sont à comprendre comme des systèmes d'expression mixte de contenus géographiques, fonctionnant par articulation d'une image à voir (la photographie dans un cas, l'espace même de la carte dans l'autre) avec un texte à lire (un titre, un commentaire, une légende), (Fontanabona, 1999). Dans les pratiques dominantes, les photographies, les carte et plus généralement les images (publicités, scénarios d'aménagement, images satellitaires) ont une fonction d'auxiliaire de la parole du professeur (Philippot \& Bouissou, 2007, Leininger-Frézal, 2014). S'agissant du texte, un aspect saillant a été travaillé, celui de la construction du sens disciplinaire de mots par ailleurs utilisés dans le sens commun qu'ils ont au quotidien. C'est le cas par exemple des notions de ville (Audigier, 1994) ou d'échange (Nonnon \& Considère, 2005). Le document composite amène par conséquent, pour la géographie, un important lot de questions touchant aux rapports intersystèmes sémiotiques.

12 Enfin, le document peut être appréhendé selon «une approche fonctionnelle et communicative [...] comme unité d'information pertinente pour un utilisateur, en fonction d'un besoin ou d'une question » (Nonnon, 2012). Autrement dit, la valeur d'un document lui est attribuée par ses utilisateurs, ici des élèves lecteurs qui s'emparant d'un support présenté à priori par le professeur comme un document, réalisent la tâche qui leur est assignée. D'un point de vue didactique, un document composite " n'est pas une voie d'accès transparente à la réalité indéniable des choses, mais un ensemble lacunaire, feuilleté, de signes dont on cherche à faire des indices pour mettre à l'épreuve des conjectures sur le réel » (ibid.).

13 Toute lecture d'un document composite nécessite de prendre en charge trois de ses propriétés : son caractère unitaire et composé ; la pluralité des systèmes sémiotiques ; enfin le rapport à un objet géographique absent physiquement, mais multi-représenté dans le document. C'est à l'élaboration d'un modèle d'analyse explorant cette problématique fonctionnelle et communicative du document composite en géographie qu'est consacrée la suite de ce texte. 


\section{Cadrage du modèle d'analyse} conduite dans des conditions normales (sans intervention d'un chercheur, hors démarche de projet) en classe de quatrième sur le thème de l'urbanisation. Cette séance est consacrée à l'étude de la ville de Détroit. Elle est préconisée pour l'enseignement du premier thème du programme, l'Urbanisation du monde : «On insiste ensuite sur la connexion des villes aux grands réseaux de la mondialisation et aux différences que cela crée entre les villes connectées et bien intégrées à une mondialisation qu'elles entrainent et des villes plus à l'écart, voire confrontées à des phénomènes de "rétrécissement" (Shrinking Cities, comme Détroit) (Ministère de l'Éducation nationale de la Jeunesse et des Sports, 2015). didacticiens les ont décrites. La première différence concerne l'origine du document support. Celui-ci n'est pas le manuel scolaire, mais un document construit par le professeur qui s'intitule : "Détroit, la "ville qui rétrécit/shrinking city" ». Une version de ce document propose à la moitié des élèves de la classe de répondre à la question: Pourquoi pouvons-nous dire que la formule "ville qui rétrécit» est justifiée? Une seconde 
version propose à l'autre moitié la question suivante : Pourquoi pouvons-nous dire que la formule "ville qui rétrécit » n'est pas tout à fait justifiée? Chaque version comprend huit éléments : deux photographies, une carte thématique et deux textes sur support papier, un photoreportage sur site web accessible par hyperlien et deux extraits de journaux télévisés accessibles par QR-code ${ }^{4}$ (voir annexe ${ }^{\circ} 1$ ).

La seconde différence concerne le scénario de la séance et le type d'interactions professeur-élèves induit. Les dossiers documentaires des manuels scolaires comportent des questions de prélèvement d'informations référées à chaque élément du dossier, puis une question de synthèse qui met en rapport les réponses préalablement obtenues. L'usage de ces dossiers donne lieu à une forme de cours dit dialogué. Le professeur pilote les activités, sollicite les élèves, les relance sur un mode de questionnement fermé tant que les réponses attendues n'ont pas été obtenues (Leininger-Frézal, 2014). Dans la séance étudiée, le professeur ne pose qu'une question (pourquoi pouvons-nous dire que...). Le scénario prévoit trois temps : la réalisation par les élèves, seuls, d'une production intermédiaire (graphe, première version d'un texte) ; la discussion de cette production avec le professeur; une réponse finale à la question posée à partir de la reprise de cette réalisation intermédiaire.

21 Pour l'enseignant, la production de textes divergents (selon que l'élève répond à la question de savoir si nous pouvons dire que la formule « ville qui rétrécit » est ou n'est pas tout à fait justifiée) alimente un temps d'institutionnalisation, à la séance suivante, du savoir associé aux «villes qui rétrécissent/shriking cities». Pour le chercheur, cette organisation permet de reconstruire à partir des textes produits par les élèves, avant le moment d'institutionnalisation, la question à laquelle ils répondent ou, pour le dire autrement, de cerner leur appropriation de la tâche définie par l'enseignant. Elle permet, dans les limites d'une approche qui ne s'attache pas aux dynamiques de la situation d'enseignement-apprentissage (interaction de régulation avec le professeur au moment de discuter la réalisation intermédiaire) d'appréhender la réception par les élèves d'un document composite à appréhender dans sa globalité. Ce que d'ordinaire, en didactique de la géographie, nous ne pouvons observer, du fait de pratiques qui segmentent la lecture documentaire et confinent le sens produit au moyen d'un guidage serré.

\section{Le modèle d'analyse}

\section{Signification}

L'analyse épistémologique nécessite de mobiliser une distinction opérée par le géographe A. Dauphiné entre l'espace terrestre : « réel et concret [...] donné, produit, vécu et perçu » (Dauphiné, 2001 [1984], p. 53) et l'espace géographique: «concept élaboré par les géographes pour formaliser scientifiquement les caractéristiques de l'espace terrestre " (ibid., p. 53). Même si la géographie scolaire a, depuis les années 1980 et en concordance avec la géographie scientifique, recentré la définition de ses contenus sur le concept d'espace (les programmes, les manuels, les pratiques, les évaluations officielles), l'usage de ce terme reste flou. Si on considère que l'espace est le concept intégrateur des contenus de géographie (Hertig, 2012), alors c'est d'espace géographique dont on parle : c'est-à-dire d'une construction intellectuelle spécifique de la discipline, mise en œuvre pour rendre compte de l'espace terrestre, surface de la 
terre, condition de l'existence des êtres humains, étendue aménagée, transformée, appropriée par les sociétés (le référent).

Cette distinction permet de poser que le document en classe de géographie sert la construction de l'espace géographique à propos de lieux et de régions du monde perçu et produit comme espace terrestre. Cette dyade doit être complétée de la notion d'espace de représentation. Géographe et épistémologue de la géographie, C. Grataloup (1993, p. 32) parle à ce sujet de "l'espace-document », qui peut être rapproché de la notion d'espace perceptuel. Il rappelle également qu'« à partir du moment où une science produit des documents, elle utilise un espace métrique banal (feuille de papier, écran...), mais orienté par nos modes généraux de représentation, à commencer par notre écriture » (ibid., p. 33). Enfin, il illustre la contrainte que cette spatialité à deux dimensions du support oppose à l'expression de contenus qui ne se réduisent pas à cette spatialité euclidienne.

Les dimensions économique et sociale des sociétés contemporaines relèvent de moins en moins du système métrique. La transposition de la distance-coût ou de la distance-temps, dans un espace document, en distance métrique est toujours un exercice intéressant, mais laisse toujours insatisfait. (ibid., p. 32).

En effet, pour beaucoup de géographes, " on ne peut définir un espace, aussi simple soit-il, sans qu'il soit l'espace de quelque chose [...] sans qu'il soit en même temps spatialité et spatialisation" (Lévy \& Lussault, 2003, p. 880). Ainsi tout contenu géographique articule une propriété spatiale (ou spatialité) faite de rapports de distance, de plus ou moins grande accessibilité des êtres et des choses, avec d'autres propriétés (économique, sociologique, démographique, culturelle, etc.) que des formes spatiales articulent. Nombre de ces propriétés font l'objet de spatialisations dont le mode de mesure n'est pas métrique. Ce qui conduit aux difficultés mentionnées par C. Grataloup concernant le système d'expression cartographique, mieux apte à représenter des distances métriques que des distances-temps, des distances-coûts, des distances affectives, culturelles, sociales, environnementales ${ }^{5}$.

Chaque fragment d'un document composite possède une matérialité spatiale (espace perceptuel) où s'expriment, en un langage donné, les propriétés de l'espace terrestre étudié. Mais le support, dans son intégralité, en contient plusieurs qui fonctionnent comme autant d'espaces de représentation. Leurs surfaces respectives, leur position relative et leur articulation (par des encadrés, par des titres, des surlignages, etc.) sur la feuille ou l'écran forment une matérialité spatiale d'échelle supérieure, dans laquelle la première est comme enchâssée. De plus en plus, cette spatialité de la représentation est elle-même matériellement composée en feuilles et/ou écrans, avec leurs divers modes d'articulation (liens internet, liens hypertexte, QR codes).

Dans notre cas, l'espace perceptuel est hybride. Le support papier recto verso comportant le titre de l'activité et la consigne qui unifient fonctionnellement le document, est prolongé de deux façons. Un hyperlien placé sous la première photographie conduit à un article en ligne édité sur le site du New York Times où le lecteur découvre un texte de présentation des travaux du photographe A. S. MacLean ainsi qu'une dizaine d'autres photographies de ce même auteur. Il est aussi associé par un $\mathrm{QR}$ Code à deux extraits de journaux télévisés de France 2 consacrés à l'évolution de la ville de Détroit. Outre le titre et la consigne, l'espace perceptuel papier est organisé par les deux photographies qui l'introduisent et le ferment. La lecture de ces deux photographies s'appuie sur les propriétés du système d'expression iconographique 
(Mendibil, 2008) : le sens à donner à l'image est fortement guidé par sa textualisation, associée à un cadrage plutôt resserré sur l'objet à voir, avec un angle de vue oblique à vertical ${ }^{6}$.

\section{Communication}

27 La théorie des signes de C. S. Peirce décrit les faits de communication humaine à partir de l'étude logique du fonctionnement des signes qu'elle mobilise. Est signe toute matérialité perçue (écrite, dessinée, photographiée, sonore, filmée) désignant une autre chose qu'elle-même pour deux personnes en communication. Dans ce cas :

un representamen [par exemple l'image visuelle ou sonore d'un mot] est le sujet d'une relation triadique avec un second appelé son objet [réel, imaginable ou inimaginable auquel le premier réfère] pour un troisième appelé son interprétant [image mentale associée à cette image visuelle ou sonore] cette relation triadique étant telle que le representamen détermine son interprétant à entretenir la même relation triadique avec le même objet pour quelque interprétant. (Peirce, 1978, p. 117). sémantique. Nous l'utilisons pour caractériser les enjeux de la communication en géographie scolaire. Dans ce cadre, la matérialité perçue (une inscription, un son, un dessin, etc.) renvoie à autre chose situé ailleurs dans l'espace terrestre. Avec le document dont nous étudions la lecture, ces rapports varient d'un fragment à l'autre, et aussi à l'intérieur d'un même fragment. Si, concernant le titre, le representamen renvoie d'un seul bloc en quelque sorte, à un objet - ce que l'on pourra aussi appeler le référent - nommé Détroit, pour certaines photographies de ce document, le référent est une parcelle, un îlot, un bâtiment, voire une partie d'agglomération dans laquelle apparait Windsor la banlieue canadienne de Détroit. Cette première marque de diversité scelle le caractère labile du référent du document composite en géographie. Mais elle se combine, dans l'acte de lecture, avec une multiplicité de rapports logiques entre representamen et référent.

31 C. S. Peirce distingue trois types de rapports signe-objet, qu'il dénomme icône, indice et symbole. L'icône entretient un rapport de similitude avec l'objet qu'il représente. Ce type de rapport se décompose en sous-types : la métaphore, le diagramme et l'image. La métaphore établit une analogie de qualité entre le signe et l'objet, le diagramme une analogie numérique et l'image une analogie de forme. L'indice, quant à lui, désigne un 
rapport de fait entre signe et objet - par exemple, la girouette et le vent. Enfin, le symbole correspond à une relation conventionnelle, arbitraire en quelque sorte.

Dans un document où plusieurs langages sont mobilisés, ces rapports sont utilisés dans des proportions diverses et selon des modalités variables. Avec le langage verbal, écrit ou oral, les rapports sont avant tout symboliques: les mots ont des rapports conventionnels avec les objets. L'image visuelle et/ou sonore de Détroit n'a pas de rapport analogique ou indiciaire avec ce qu'elle désigne. Néanmoins, le rapport métaphorique peut être exploité, comme avec cette formule d'un des composants textuels : «Détroit, dont la superficie couvre l'équivalent de San Francisco, Manhattan et Boston réunis, ressemble encore à une ville gruyère [nous soulignons] avec d'étranges îlots de verdure autour desquels subsistent des ruines fantomatiques ». Les îlots de verdure sont à la ville de Détroit ce que les trous sont à un morceau de gruyère. La dénomination de Rust Belt (ceinture de rouille) qui désigne ordinairement en géographie la région urbaine du Nord-Est des Etats-Unis touchée depuis les années 1970 par la désindustrialisation, la paupérisation et la dépopulation exploite le même rapport. Elle n'est pas sans tendre vers un rapport indiciaire par lequel le lecteur s'attend à voir (et donc voit) des villes-usines à l'abandon. Nous reviendrons dans l'analyse sur le problème de compréhension que pose la formule incluse dans le titre : la ville qui rétrécit, traduction française de la catégorie Shrinking City.

La cartographie emprunte largement à l'image (toujours au sens de C. S. Peirce) pour les formes et les tracés représentés, quelquefois au diagramme par exemple pour l'épaisseur des traits qui représentent les routes dans la carte intitulée «carte de l'occupation du sol dans la ville de Détroit ». En revanche, sur cette même carte, les figurés représentant les taux d'abandon relèvent du symbole. Ce qui n'est pas le cas des aplats de couleur verte qui représentent des espaces verts sur la carte du second support documentaire. Le choix du figuré joue alors de la relation indiciaire qui souvent oriente la lecture d'une carte, faisant d'elle le reflet du réel en vue zénithale (Fontanabona, 2000). L'iconographie photographique fait fonctionner simultanément des relations d'analogie et d'indice pour la part iconique du support: la perception visuelle d'une portion d'espace assure le lecteur de l'existence de ce fragment de réel. Il arrive que la textualisation de l'image (un titre, un commentaire, une légende) fasse jouer des rapports différents, en particulier métaphoriques ; ce qui n'est pas le cas dans ce dossier.

$\mathrm{Au}$ caractère labile du référent représenté, la multiplicité de rapports logiques institués entre représentamen et référent, diversement exploités en fonction des langages, vient donc ajouter de la complexité à la communication.

\section{Expression}

La théorie linguistique de L. Hjelmslev est utile pour appréhender la façon dont les langages mobilisés en classe de géographie mettent en forme les contenus disciplinaires. Ce linguiste substitue à la dyade signifiant-signifié, la dyade expressioncontenu. De plus, il distingue dans chacun de ces deux plans la forme et la substance ; la forme étant ce qui découpe, articule, organise une substance par elle-même amorphe. Sur le plan de l'expression, la forme organise, articule une substance sensible, perceptible. Appliquée à son domaine originel d'efficience, la forme de l'expression correspond aux règles phonologiques propres à une langue, qui, à partir du continuum 
des sons qu'un appareil vocal humain peut produire, déterminent un nombre limité de phonèmes et les relations qui les unissent. Sur le plan du contenu, la forme correspond au découpage en unités de sens ou en catégories, d'une substance organisable autrement. Pour Hjelmslev, « tout système de figures au service des signes renferme en soi une forme de l'expression et une forme du contenu» (Hjelmslev, 1976 [1943], p. 77). Le signe est « donc à la fois signe d'une substance du contenu et signe d'une substance de l'expression » (ibid., p. 76).

Articulée aux catégories que l'épistémologie de la géographie a construites, sur le plan du contenu, la forme correspond au découpage en catégories spatiales, d'une substance sociale organisable autrement: en termes d'économie, de structuration sociale, de culture, de démographie, etc. Ces considérations ne sont pas sans intérêt pour comprendre la complexité des rapports sémiotiques installés dans un même document. Prenons un contenu parmi d'autres dans le cas que nous analysons: l'évolution des fonctions urbaines dans une "ville qui rétrécit/shrinking city». Il s'agit d'un phénomène économique dont la spatialité se manifeste par des changements d'occupation du sol, en divers lieux caractérisés par l'abandon et une agriculture urbaine de reconquête.

Le document propose quatre occurrences d'articulation formelle -forme de l'expression et forme du contenu - d'une substance que l'on peut qualifier de "verte » sur le plan de l'expression (le vert comme un segment que l'on découpe dans le spectre continu des couleurs) et qui, sur le plan du contenu, a à voir avec cette évolution des fonctions urbaines ou de l'occupation du sol urbain caractéristique des villes américaines en décroissance.

Une première occurrence apparaît avec le titre de la photographie "un jardin communautaire urbain cultivé à la place des maisons abandonnées ", lequel renvoie dans l'espace iconique de représentation, à la vue en oblique de rangées de végétaux cultivés et de tunnels de culture, au sein de parcelles que délimitent et découpent des routes urbaines. En somme, une substance sensible verte (plutôt des teintes de vert, du bleuté ainsi que le gris des tunnels de culture) mise en forme par les lignes des tunnels et le damier des routes (forme de l'expression). Et, du côté du contenu, pris en charge d'abord par le titre, une substance de changement qu'exprime "à la place de " pour une forme spatiale qui est celle de la parcelle ou du sous-quartier.

Une deuxième occurrence est produite cette fois-ci par un court texte journalistique où est décrite une "ville gruyère avec d'étranges îlots de verdure autour desquels subsistent des ruines fantomatiques». Formule qui fait de la catégorie verdure, la structure élémentaire d'une "ville gruyère» (une forme spatiale organisant la substance d'une économie en mutation).

Une troisième occurrence concerne une carte de l'occupation du sol à Détroit où la légende indique des "espaces verts» (forme de contenu fonctionnel) représentés au moyen d'aplats de couleur verte (forme de l'expression). Cependant le contenu géographique ne renvoie que de façon très incertaine au sujet. Il est probable qu'une partie de ces « espaces verts » (parcs et jardins publics) ne soient pas nés de la crise que connaît Détroit. Le document ne permet pas de le savoir, mais pour des élèves, ces « espaces verts" peuvent être assimilés aux «îlots de verdure» dont parle le texte précédent. 
41 La dernière occurrence ramène le lecteur à l'iconographie. Au premier plan de la photographie ainsi commentée : « Le projet Heidelberg avec ses maisons colorées et ses installations artistiques à partir d'objets de récupération attire 70000 personnes par an ", un morceau de pelouse, vert éclatant sous le ciel bleu (forme de l'expression), vient, sans soulignement textuel, signer en quelque sorte une possibilité de renouvellement urbain par la culture à Détroit (forme du contenu).

Qu'est-ce que lire et comprendre un document composite pour s'approprier des savoirs géographiques? Le modèle élaboré retient qu'il s'agit 1) d'une activité d'organisation de contenu, usant de systèmes d'expression diversement aptes à l'articulation de spatialités qui caractérise l'approche disciplinaire (géographique) du social ; 2) à propos d'un référent représentable de façon multiple et non nécessairement concordante bien que désigné souvent d'une manière unifiée (un toponyme) ; 3) avec lequel ces systèmes d'expression jouent de rapports sémiotiques multiples dans un espace perceptuel, luimême unitaire et composé.

\section{Quelques observations sur un cas de lecture de document composite}

Le réalisme qui gouverne la production de savoir en géographie scolaire s'alimente à des pratiques de guidage serré de l'interrogation sur des documents en petit nombre pris successivement. Elles déchargent les élèves de toute responsabilité interprétative dans la saisie d'ensemble d'un document composite. Mais, dans le cas que nous étudions, à la différence des pratiques ordinaires, les élèves se sont vu confier la responsabilité de donner un sens au document composite qui leur était soumis. Quand un professeur leur dévolue cette responsabilité, comment des élèves s'en saisissent-ils? Qu'est-ce que notre modèle d'analyse nous donne à voir des difficultés auxquelles ils font alors face dans la lecture du document? L'objectif n'est pas de conduire une analyse de corpus, mais d'aborder, à l'aide de quelques extraits de textes d'élèves, leur "gestion" des problématiques que le modèle soulève: parviennent-ils (ou non) à articuler des spatialités multiples (démographiques, économiques, culturelles, etc.), à propos d'un «objet» à la fois donné de toute évidence (Détroit) et représenté de manière multiple, par les points de vue et par les systèmes sémiotiques?

Trois types de textes ont été produits par les élèves. Ils correspondent à autant de façons de s'approprier la tâche proposée. Ils peuvent aussi se définir comme des formes de résolution du problème qu'il leur a été posé, à savoir élaborer un contenu géographique valide, à partir d'un ensemble fragmenté, démultiplié « de signes dont on cherche à faire des indices" et convergeant vers une réponse fondée, concise et unitaire.

Le premier type de texte résulte d'une activité de mise en ordre d'informations récoltées dans les fragments écrits du document ${ }^{7}$.

Je pense que nous pouvons dire que la formule « ville qui rétrécit » n'est pas tout à fait justifiée car même si la moitié de la population est partie, il y a quand même encore des travaux urbains (la ligne de tramway et les maisons colorées). Puis il y a beaucoup de touristes qui viennent visiter la ville (Thomas).

La ville rétrécit depuis 2007 avec la chute des entreprises, les Blancs qui partent dans les banlieues. Il reste encore le centre-ville habité, mais tout le reste est inoccupé, abandonné. Donc oui, la ville de Détroit rétrécit, mais peut-être que cela changera (Léo). 
èves procèdent en rapprochant des indices d'une attractivité perdue, maintenue ou retrouvée de la ville, avec la connotation qu'ils donnent au mot " rétrécissement ", celle d'une perte (ou non) de centralité. La ville est ici conçue comme un tout dont l'évolution, à la hausse ou à la baisse, se traduirait par une forme spatiale en extension ou en rétraction. Les élèves ont mis en œuvre un procédé typique de la pensée sociale laquelle se saisit de la catégorie de ville, dans la continuité des pratiques, comme d'un lieu central, dense et actif. Dès lors, les élèves sélectionnent des informations qu'ils peuvent traiter selon une grille attraction/répulsion, concentration/dilution, extension/rétraction. La métaphore explicite de la ville gruyère n'a pas été retenue, pas plus que les photographies pouvant faire indice d'un évidement de l'espace habité. Ce n'est pas tant l'enjeu de la catégorisation d'une ville qui les focalise qu'un diagnostic général sur l'état de celle-ci.

Dans un deuxième type de texte, les élèves mettent en place un récit dont le protagoniste principal est Détroit.

Il fut un temps où Détroit rétrécissait, mais c'est du passé. Six ans après s'être déclarés en crise économique, les hôtels connurent un afflux de touristes venus admirer les ruines de Détroit [...] Maintenant Détroit est pleine d'effervescence et compte 80000 habitants (Clément).

Détroit est en pleine effervescence. La première ligne de tramway de la ville a été inaugurée. La ville a mis aux enchères des maisons [...] Les grands constructeurs automobiles ont quand même gardé leurs centres de recherche. Détroit a enrayé l'hémorragie sociale et économique (Tom).

Les élèves sélectionnent des informations expressément référées à Détroit, mais associées à des contenus différents :

- un contenu morphologique avec « la première ligne de tramway de la ville »;

- un contenu de localisation avec «les grands constructeurs automobiles ont quand même gardé leurs centres de recherches à Détroit »;

- un contenu d'acteur de l'aménagement, « la ville a mis aux enchères des maisons ».

Le procédé de personnification utilisé au début et à la fin du texte permet de réduire la polyphonie du récit: "Détroit est en pleine effervescence»; "Détroit a enrayé l'hémorragie sociale et économique». Le texte se rattache à un genre du récit que l'enseignement de thèmes d'aménagement conforte en géographie (Leininger-Frézal \& Carré, 2016). Ce n'est pas non plus la catégorie de rétrécissement qui est l'objet du travail, mais la trajectoire d'une entité, presque d'un être géographique.

Le troisième type de texte correspond mieux à l'objectif de la tâche proposée par le professeur, à savoir discuter la valeur de la catégorie « ville qui rétrécit/Shrinking City ».

En bref, Détroit n'est pas une ville qui rétrécit mais qui avait rétréci en sachant qu'une ville ne rétrécit pas en dimension (étendue, surface), mais plutôt elle diminue (le nombre) (Lucie).

Je pense que Détroit rétrécit dans le sens où la ville perd de la population ainsi que de la surface à cause des maisons détruites et des immeubles rasés. Je pense que Détroit rétrécit tout en gardant les mêmes frontières, les mêmes limites (Loïsse).

51 Les deux élèves soulignent l'ambiguïté du terme, ce qui nécessite qu'elles aient pris en compte des contenus mis en forme non seulement par les textes comme dans les cas précédents, mais aussi par les photographies et la cartographie. Sans formuler ces notions d'ailleurs absentes des éléments textuels du document composite, elles identifient une "perforation urbaine» (Florentin, Fol \& Roth, 2009) et un « surdimensionnement » du tissu urbain par rapport au nombre actuel de ses habitants 
(Florentin, 2016). Cela suppose de dissocier espace et substance sociale, c'est-à-dire, à la différence des élèves auteurs du premier type de texte, de ne pas faire de l'espace le reflet fidèle des évolutions sociales, mais de lui reconnaître une autonomie de fonctionnement, avec laquelle interagissent les acteurs sociaux. Cela suppose également, à la différence des élèves auteurs du deuxième type de texte, de dissocier spatialité de la forme urbaine et spatialité de la démographie ainsi que de l'économie, de ne pas les subsumer sous la catégorie d'un " être urbain » doué d'une vie autonome (Détroit).

\section{En guise de conclusion}

La lecture de document composite confronte assurément les élèves à la difficulté de construire un questionnement permettant de lui attribuer un sens (Nonnon, 2012). Si le professeur a installé des signes d'unité : un titre et une consigne en haut d'un espace perceptuel rassemblant les types de composants attendus d'un document composite en géographie, les élèves n'en ont pas moins fortement divergé dans l'appropriation de la question qui leur était posée.

Même succincte, la présentation de cette diversité de textes nous permet de formuler des difficultés auxquelles les élèves ne sont en général pas confrontés du fait du guidage serré des activités avec le document en géographie scolaire. Notre modèle d'analyse permet d'en cerner trois qui correspondent à autant d'enjeux d'appropriation de la pensée disciplinaire, dès lors que les élèves ont à exercer la responsabilité de l'interprétation d'un document composite et de la consigne qui l'accompagne.

1. Un premier enjeu est la "gestion » des possibilités d'expression de la dimension spatiale du contenu géographique, variables selon les langages utilisés dans le document et à utiliser pour répondre à la consigne du professeur. Ici, le fait que la production finale doive emprunter au langage verbal écrit a rendu difficile l'expression d'une spatialité caractérisée par l'évidement, la fragmentation et l'entre-deux, spatialité que l'iconographie et la cartographie, par analogie de formes, expriment mieux. En revanche, le langage verbal écrit est mieux à même de prendre en charge les contenus démographiques et économiques. Si la production finale avait été demandée sous la forme d'un croquis, la lecture du document composite aurait sans doute été différente ;

2. Un deuxième enjeu concerne la « gestion » des prises offertes par le document à une lecture dans le registre de la pensée sociale. Celle-ci réduisant la complexité des processus en référant à l'expérience non scolaire des élèves (Considère, 2017), l'enjeu de son contrôle disciplinaire est variable en fonction des thèmes d'étude et des langages utilisés. Dans le cas étudié, si les éléments textuels avaient été moins fournis en informations factuelles, en qualificatifs et surnoms attribués à Détroit, alors peut-être l'image mentale la ville (l'interprétant dans la théorie de C.S. Peirce) ainsi produite ne serait pas entrée aussi facilement en résonance avec une pensée sociale qui réduit cette ville - ou toute autre ville à un objet saisi depuis son centre ou à un être doué d'une vie autonome ;

3. Un troisième enjeu est la prise en compte de la multiplicité des points de vue représentés dans le document pour répondre à la question posée. Pour réaliser la tâche, les élèves doivent trouver une forme de réduction de cette polyphonie. Les deux premiers types de textes correspondent à des formes appuyées de réduction, classiques en géographie scolaire : le classement d'informations factuelles décontextualisées, l'« animation » d'entités qui deviennent de quasi-personnages. Le troisième type de texte parvient à ouvrir un «espace de discussion» parce qu'il prend pour objet la catégorie d'analyse "villes qui rétrécissent/shrinking cities» ('’espace géographique dans notre modèle d'analyse) et non le 
référent qu'elle sert à décrire (l'espace terrestre dans notre modèle d'analyse), ainsi qu'il est de coutume dans une géographie scolaire massivement réaliste.

54 Cet ensemble non exhaustif de sources de difficultés, non repérées dans les prescriptions et recommandations en géographie, invite au repérage de variables permettant d'étalonner des niveaux de difficulté, de proposer des progressions d'apprentissage de la lecture de document composite en géographie, enfin d'étayer ces progressions par des recherches mettant en œuvre des protocoles comparatifs (par contenu d'apprentissage, composition des documents, représentation des systèmes d'expression dans le document, référent géographique, etc.).

\section{BIBLIOGRAPHIE}

AUDIGIER, F. (1993). « Documents en classe : traditions, mythes et réalités ». Actes du $7^{e}$ Colloque de didactiques de l'histoire, de la géographie, des sciences sociales. Documents : des moyens pour quelles fins? Paris : INRP, p. 13-24.

AUDIGIER, F. (1994). « Des élèves, des villes. Représentations et didactique ». Revue de Géographie de Lyon 69 (3), p. 205-219. En ligne : https://www.persee.fr/doc/ geoca_0035-113x_1994_num_69_3_4257.

AUDIGIER, F. (1996). Recherches de didactiques de l'histoire, de la géographie, de l'éducation civique. Notes de synthèse pour le diplôme d'habilitation à diriger des recherches, Université Diderot Paris VII.

CONSIDÈRE, S. (2017). Représentations des élèves et apprentissages en géographie. Mémoire d'habilitation à diriger des recherches : Université de Lille 3.

DAUPHINÉ, A. (2001) [1984]. « Espace terrestre et espace géographique ». In : Bailly A. (éd.). Les concepts de la géographie. Paris/New York/Barcelone : Masson, p. 43-54.

FLORENTIN, D. (2016). « Notion en débat : shrinking city ». Géoconfluences ENS Lyon. Mis en ligne le 16 nov. 2016. En ligne : http://geoconfluences.ens-lyon.fr/informations-scientifiques/a-la-une/ notion-a-la-une/notion-shrinking-city.

FLORENTIN, D., FOL, S. \& ROTH, H. (2009). « La "Stadtschrumpfung” ou "rétrécissement urbain" en Allemagne : un champ de recherche emergent ». Cybergeo. European Journal of Geography, document 445. En ligne : https://journals.openedition.org/cybergeo/22123.

FONTANABONA, J. (1999). « Mieux comprendre comment un élève donne du sens aux cartes ». Cahiers de Géographie du Québec, 43 (120), p. 517-538. En ligne : https://www.erudit.org/fr/revues/ cgq/1999-v43-n120-cgq2692/022853ar/.

FONTANABONA, J. (dir.) (2000). Cartes et modèles graphiques. Analyses de pratiques en classe de géographie. Paris : INRP.

FRÉMONT, A. et al. (1982). Espaces vécus et civilisations. Paris : CNRS Éditions.

GENEVOIS, S. (2008). Quand la géomatique rentre en classe. Usages cartographiques et nouvelle éducation géographique dans l'enseignement secondaire. Thèse en géographie : Université Jean Monnet SaintEtienne. En ligne : https://tel.archives-ouvertes.fr/tel-00349413/document. 
GENEVOIS, S. (2016). « Outils géomatiques et apprentissages en géographie : quels enjeux du point de vie de la recherche en éducation? ». Les Sciences de l'éducation. Pour l'Ère nouvelle 49 (4), p. 93-116. En ligne : https://www.cairn.info/revue-les-sciences-de-l-education-pour-l-erenouvelle-2016-4-page-93.htm/.

GRATALOUP, C. (1993). « Les moyens de l'imaginaire du géographe : modes de représentation du monde et de la discipline ». Actes du $7^{e}$ Colloque de didactiques de l'histoire, de la géographie, des sciences sociales. Documents : des moyens pour quelles fins? Paris : INRP, p. 29-39.

HERTIG, P. (2012). «Didactique de la géographie et formation initiale des enseignants spécialistes. Conception et première évaluation du nouveau dispositif de formation initiale des enseignants de géographie du Secondaire supérieur à la HEP Vaud », Géovisions 39. Lausanne : Institut de géographie de l'Université.

HJELMSLEV, L. (1975) [1948]. Prolégomènes à une théorie du langage. Paris : Hatier.

LEBRUN, M. \& LACELLE, N. (2012). « Le document multimodal : le comprendre et le produire en classe de français », Repères 45, p. 81-95. En ligne : https://journals.openedition.org/reperes/141.

LECLAiRe-HALTÉ, A. \& MAISONNEUVE, L. (2018). Appel à contribution : Lire des documents composites en classe. En ligne : https://www.afef.org/appel-contribution-lire-des-documents-composites-enclasse.

LEFORT, I. (1992). La lettre et l'esprit. Géographie scolaire et géographie savante en France, 1870-1970. Paris : CNRS Éditions.

LEININGER-FRÉZAL, C. (2014). « Une rhétorique des images du risque dans la géographie scolaire ». Mappemonde 113 (1). En ligne : http://mappemonde-archive.mgm.fr/num41/articles/ art14103.html

LEININGER-FRÉZAL, C. \& Carré, C. (2016). « La géographie scolaire : d'un tournant actoriel à l'autre ». In : Thémines, J.-F. \& Doussot, S. (dirs). Acteurs et action. Perspectives en didactiques de l'histoire et de la géographie. Caen : Presses universitaires de Caen, p. 227-243.

LÉVY, J. \& LuSSAULT, M. (dirs) (2003). Dictionnaire de géographie. Paris : Belin. MARGAIRAZ, D. (1988). «L'utilisation des supports informatifs dans l'apprentissage de l'histoire et de la géographie ». Revue française de pédagogie 85, p. 29-36. En ligne : https://www.persee.fr/doc/ rfp_0556-7807_1988_num_85_1_1434.

MENDIBIL, D. (2008). « Dispositif, format, posture : une méthode d'analyse de l'iconographie géographique ». Cybergeo. European Journal of Geography, document 415. En ligne : http:// journals.openedition.org/cybergeo/16823.

Ministère de l'Éducation nationale de la Jeunesse et des Sports (2015). Bulletin officiel spécial $n^{\circ} 11$ du 26 novembre 2015. En ligne : https://www.education.gouv.fr/pid285/bulletin_officiel.html? pid_bo=33400.

NONNON, É. (2006). «Apprendre des mots, construire des concepts : les termes de l'échange en géographie ». In : Garcia-Debanc, C. \& Plane, S., Le Lexique et son apprentissage. Paris : Hatier.

NONNON, É. (2012). « Dimension épistémique de la lecture et construction de connaissances à partir de l'écrit : enjeux, obstacles, apprentissages », Repères 45, p. 7-37. En ligne : https:// journals.openedition.org/reperes/132.

NONNON, É. \& CONSIDÈRE, S. (2005). «Les tensions entre énonciation orale et notation schématique : exemples d'activités impliquant la représentation du point de vue et de relations orientées (la 
notion d'échanges au Cours Moyen) ». In : Weil-Barais, A. (dir.), Noter pour penser. Approches développementales et didactique. Actes du colloque d'Angers, 27-28 janv. 2005.

PHILIPPOT, T. \& BouISSOU, C. (2007). «Les images en géographie : qu'en font les enseignants et les élèves ? » SPIRALE. Revue de Recherches en Éducation 40, p. 37-49. En ligne : https://www.persee.fr/ doc/spira_0994-3722_2007_num_40_1_1392.

PEIRCE, C.S. (1978). Écrits sur le signe. Trad. de l'anglais et éd. par G. Deledalle. Paris : Éditions du Seuil.

THÉMINES, J.-F. (2002). « Propositions pour un transfert du concept de rapport au savoir en didactique de la géographie ", Cahiers de la MRSH 28, p. 35-56.

TUTIAUX-GUILLON, N. (2004). L'histoire-géographie dans le secondaire. Histoire d'une inertie scolaire. Habilitation à diriger des recherches : Université Lumière Lyon 2.

\section{Sources utilisées dans le document composite en annexe :}

ANIZON, E. (2010). «Detroit, la ville qui rétrécit ». Télérama. Mis en ligne le 17 déc. 2010. En ligne : https://www.telerama.fr/monde/peut-on-sauver-detroit,63724.php.

FONTAN, S. (2014). « La faillite de la ville de Détroit aux États-Unis : triomphe et déclin ». Économie Matin. Mis en ligne le 8 juin 2014. En ligne : http://www.economiematin.fr/news-detroit-failliteville-usa-crise-abandon-population.

DANGerfield, W. LOUTtit, M. \& HENSON SCALES, J. (s.d.) « Detroit by Air. Photographs by Alex S. MacLean ». The New York Times. En ligne : https://www.nytimes.com/interactive/2014/12/07/ opinion/sunday/exposures-detroit-by-air-alex-maclean.html? mtrref=www.google.com\&assetType=REGIWALL.

Detroit Future City (2012). «The Land Use Element. The Image of the City ». En ligne : https:// detroitfuturecity.com/wp-content/uploads/2014/02/DFC_LandUse_2ndEd.pdf.

\section{ANNEXES}

\section{Annexe 1}



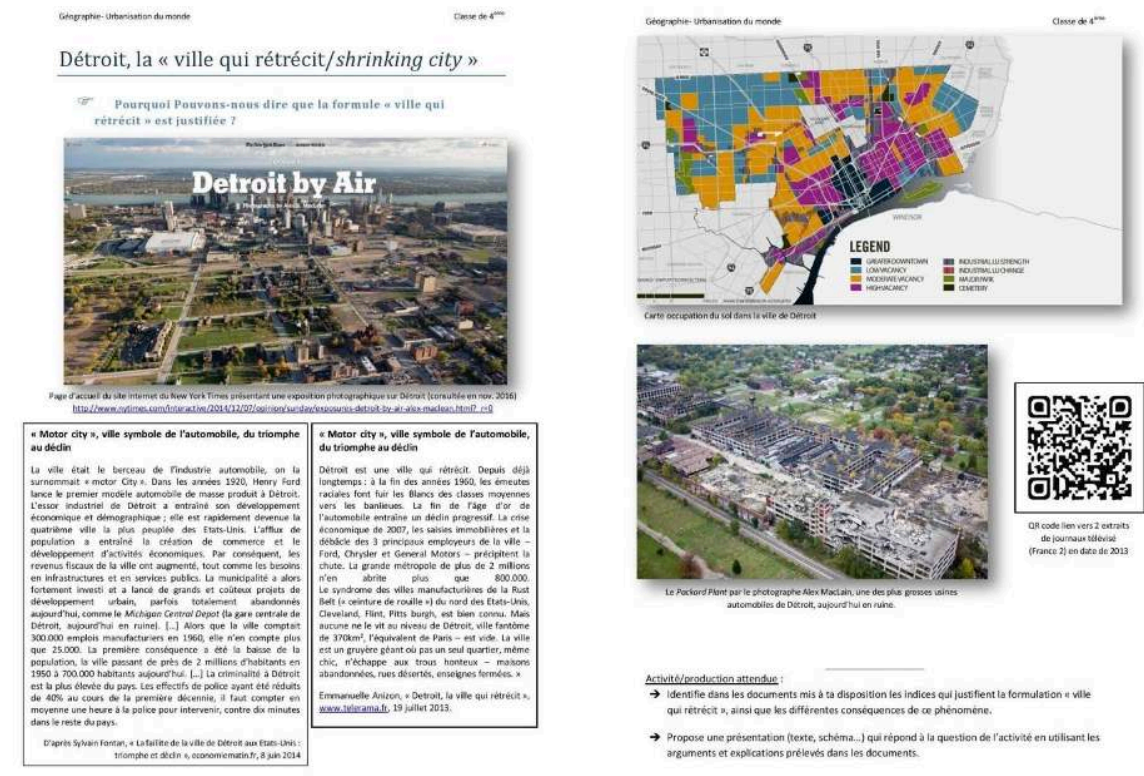

\section{NOTES}

1. Dans l'histoire de ces didactiques qui débute à la toute fin des années 1980 .

2. La géomatique désigne l'utilisation professionnelle (en aménagement, urbanisme, géomarketing, archéologie, géographie, etc.) de technologies numériques pour acquérir, traiter, visualiser et communiquer de l'information géographique.

3. Ces trois aspects ne couvrent pas l'ensemble des propriétés d'une situation de lecture de document en géographie. Sont exclus de cette modélisation, par exemple, les aspects de navigation et de mobilité dans la découverte du document (Genevois, 2008) ou les aspects d'engagement du corps et de sollicitation des sens, en particulier tactile et plus précisément haptique.

4. Ce type de composition documentaire est appelé par son auteur professeur un document augmenté. Nous remercions chaleureusement N.Cordray, professeur d'histoire-géographie et formateur académique pour avoir accepté de nous communiquer le corpus analysé. Il est l'auteur du document composite reproduit en annexe $n^{\circ} 1$.

5. Ces modes de mesure de la distance, ces types de distance sont identifiées dès les années 1970 (Frémont et al., 1982) et ne cessent depuis d'alimenter les travaux de géographie sociale et culturelle

6. Le cadrage resserré et les angles de vue oblique à vertical ont été construits par la tradition iconographique de la géographie scientifique et scolaire en mode de représentation objectivant. La stratégie iconographique qui consiste à imposer un titre désignant ce qui est à voir produit un «confinement du sens » de l'image (Mendibil, 2008). 
7. Les citations sont des extraits de la version finale des textes d'élèves, extraits situés à la fin de ces productions qui, généralement, ont une longueur d'une page à une page et demie.

\section{RÉSUMÉS}

Qu'est-ce que lire et comprendre un document composite en classe de géographie ? À quelles difficultés propres à ce type de document les élèves sont-ils confrontés pour interpréter le monde tel qu'il est conçu dans cette discipline scolaire? Cet article propose un modèle épistémologique et sémiotique pour identifier certaines de ces difficultés. Elles sont illustrées avec un cas d'étude des villes qui rétrécissent en classe de quatrième.

What is reading and understanding a composite document in geography? What difficulties are students facing in interpreting the world as it is conceived in this school discipline? The paper proposes an epistemological and semiotic model to identify some of these difficulties. They are illustrated with a case study of shrinking cities, in year $9 / 8^{\text {th }}$ grade class.

\section{INDEX}

Mots-clés : document, document composite, didactique de la géographie, géographie scolaire Keywords : document, composite document, teaching of geography, school geography.

\section{AUTEUR}

JEAN-FRANÇOIS THÉMINES

Inspé Normandie Caen/Université de Caen Normandie, ESO, F-14032 Caen, France 